\title{
Practical Management Recommendations for Anti-ErbB2 Therapy with Lapatinib
}

\author{
Dimitrios Chatsiproios $^{a} \quad$ Renate Haidinger $^{b} \quad$ Thomas Suter $^{c}$ \\ a CGG Klinik GmbH, Mannheim, \\ ${ }^{\mathrm{b}}$ Brustkrebs Deutschland e.V., München, Germany \\ ${ }^{c}$ Klinik und Poliklinik für Kardiologie, Schweizer Herz- und Gefäßzentrum Bern, Universitätsklinik, Inselspital, Bern, Switzerland
}

\section{Introduction}

The toxicity profiles of conventional cytotoxic regimens are nowadays well established. Well known adverse events comprise myelotoxicity, mucositis, nausea and vomiting, alopecia, fatigue, neuro- and cardiotoxicity, which can be life-threatening and/or unacceptable for the individual patient.

Novel targeted therapies are designed in the effort to enhance anti-tumor activity by interfering with specific signaling pathways crucial for proliferation, survival, and angiogenesis of the tumor. Presumably, specific targeting of these pathways also decreases the aforementioned toxicities usually observed with conventional chemotherapy which unselectively hits all proliferating cells. Generally, monoclonal antibodies and small molecules targeting the ErbB family, which are now increasingly used in clinical practice, are well tolerated. However, it is important to point out that the same signaling pathways that are targeted in cancer cells are also important for cell survival in other tissues. Indeed, many of the signaling pathways that are or will be inhibited in cancer patients are targets that need to be stimulated in other cell types. Well known examples in cardiology are the ErbB2/NRG (neuregulin) system which needs to be stimulated in failing hearts, and the vascular endothelial growth factor (VEGF) which is already stimulated in cardiovascular patients with arteriosclerosis $[1,2]$. Thus, by blocking pathways in normal body cells, a host of different adverse reactions can emerge with some of the newer cancer drugs especially diarrhea, toxicity of the skin and nails, cardiotoxicity, and pigment disorders. As most of the new biologicals are used in combination with cytotoxics, potential interactions leading to increased toxicities also have to be considered.

\section{Safety Profile of Lapatinib}

Lapatinib is an orally available tyrosine kinase inhibitor, blocking both EGFR/ErbB1 and HER2/ErbB2. There are considerable data from several trials concerning the adverse effects and safety profile of this small molecule, which suggest that the incidence of adverse reactions is lower than that observed with many cytotoxics, and that the rate of severe toxicities (grade 3 and 4) does not exceed 3-4\%.

In the pivotal phase III trial EGF 100151, lapatinib in combination with capecitabine was compared with capecitabine alone in women with locally advanced or metastatic ErbB2overexpressing breast cancer who had progressed after chemotherapy and trastuzumab [3]. With the exception of diarrhea and rash, the rates of most adverse events (hand-foot syndrome, nausea, vomiting, fatigue) were comparable between the two arms. In the combination arm $29 \%$ of women versus $15 \%$ in the capecitabine monotherapy arm developed rash, which was mostly mild or moderate. Sixty-five \% of patients in the combination therapy group experienced diarrhea versus $40 \%$ of the capecitabine-treated patients. Most cases of diarrhea were mild or moderate.

The safety profile of lapatinib was confirmed by the results of the phase III trial EGF 30001, which compared the combination of lapatinib with paclitaxel versus paclitaxel alone as firstline therapy in patients with locally advanced or metastatic breast cancer overexpressing ErbB2 [4]. Diarrhea was the most common adverse event experienced by $58 \%$ of women in the combination arm and $26 \%$ of patients in the paclitaxel monotherapy arm. Unfortunately, 3 patients in the combination group had fatal events of diarrhea and septic shock in the initial phase of the trial, which might have been related to a lack of experience in the management of lapatinib-associated diarrhea. A second related factor could be a pharmacokinetic interaction between lapatinib and paclitaxel, leading to an

\begin{tabular}{|c|c|}
\hline KARGER & (C) 2008 S. Karger GmbH, Freiburg \\
\hline $\begin{array}{l}\text { Fax +49761 } 4520714 \\
\text { E-mail Information@Karger.de } \\
\text { www.karger.com }\end{array}$ & $\begin{array}{l}\text { Accessible online at: } \\
\text { www.karger.com/brc }\end{array}$ \\
\hline
\end{tabular}




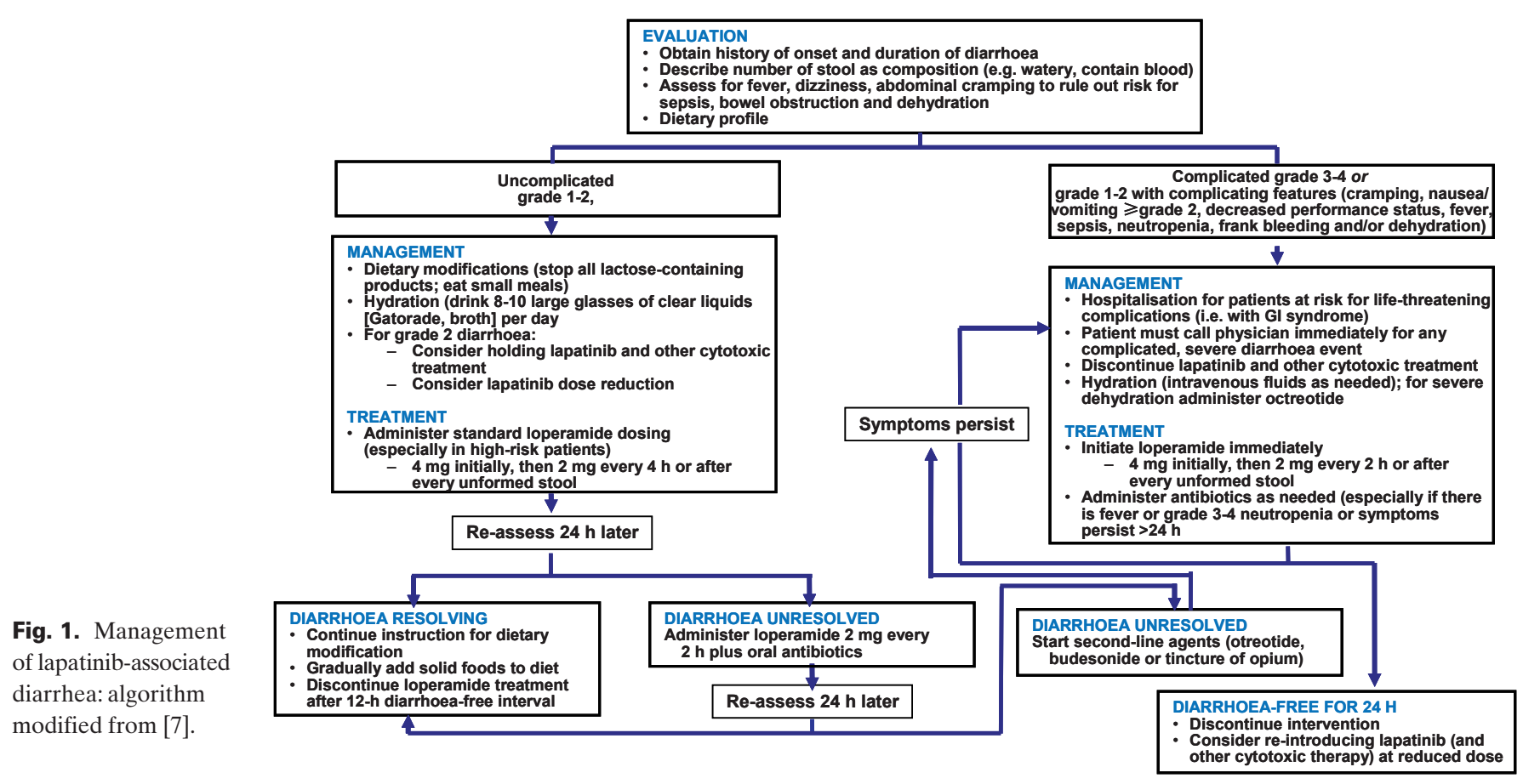

increase of about $20 \%$ in the area under the curve (AUC) of both drugs [5]. The second most common adverse reaction in this study was rash in $44 \%$ of women in the combination group and $22 \%$ of women treated with paclitaxel only.

\section{Clinical Management of Diarrhea}

In their analysis of nearly 2,100 cancer patients treated with lapatinib alone or in combination with capecitabine, Koehler et al. [6] described an incidence of diarrhea of 47 and $61 \%$, respectively. Diarrhea was mostly mild to moderate and often did not require intervention. Nevertheless, adequate monitoring of the patients is crucial. In most cases (>90\%), diarrhea appears early, during the first days of treatment (before day 6) and is of short duration (median 5 days). Most diarrhea events associated with lapatinib treatment can be managed by observation only, without the need for dose interruption, dose adjustment, or discontinuation of therapy. Most patients who require intervention respond to standard antidiarrheal medications (loperamide), which should be prescribed upfront together with lapatinib. Patients should be encouraged to keep dietary diaries in order to detect other medications and dietary supplements potentially interacting with lapatinib. Only in rare cases of severe diarrhea, rehydration, octreotide (not licensed in Germany for this indication), or antibiotics are warranted.

Benson et al. [7] have developed a useful algorithm for the management of lapatinib-associated diarrhea and stress the importance of a careful evaluation of patient history (dietary profile) as well as daily monitoring of symptoms. In cases of uncomplicated diarrhea, these authors advise sufficient drinking of broth or isotonic beverages and a lactose-free diet (fig. 1). Withdrawal of lapatinib is only recommended in patients not responding to loperamide. Re-initiation of lapatinib can be considered in patients experiencing no diarrhea over a 24-h period.

\section{Clinical Management of Skin Rash}

About half of the patients exposed to lapatinib in 8 clinical trials experienced skin events, mostly papules and pustules on the back and chest, rarely in the face [8]. Hair disorder, dry skin, and pruritus or urticaria were rare (3-4\%); nail disorders and infections were reported in less than $1 \%$ of patients. As diarrhea and skin reactions are normally of low grade (1 or 2), they usually do not require interventions, dose reductions, or discontinuation of lapatinib. In an overview by Sweetman et al. [8], the frequency of severe skin reactions of grade 3 was low $(6 \%)$, with grade 4 reactions not reported. Most of the skin events developed early, in the first 2 weeks of treatment. The majority $(87 \%)$ of skin disorders resolved spontaneously within 3-4 weeks. Skin rash is known as a class effect of drugs targeting ErbB1; however, the incidence of this adverse reaction is lower during lapatinib treatment compared to other ErbB1 inhibitors [9].

For the management of skin rash it is essential to differentiate between papular and pustular eruptions, and to consider the extent of body surface affected, as well as the severity of symptoms. Treatment consists of topical application of corticosteroids or metronidazole in mild cases, and oral corticos- 
Fig. 2. Management of lapatinib-associated rash: algorithm modified from [10]. BSA $=$ Body surface area.

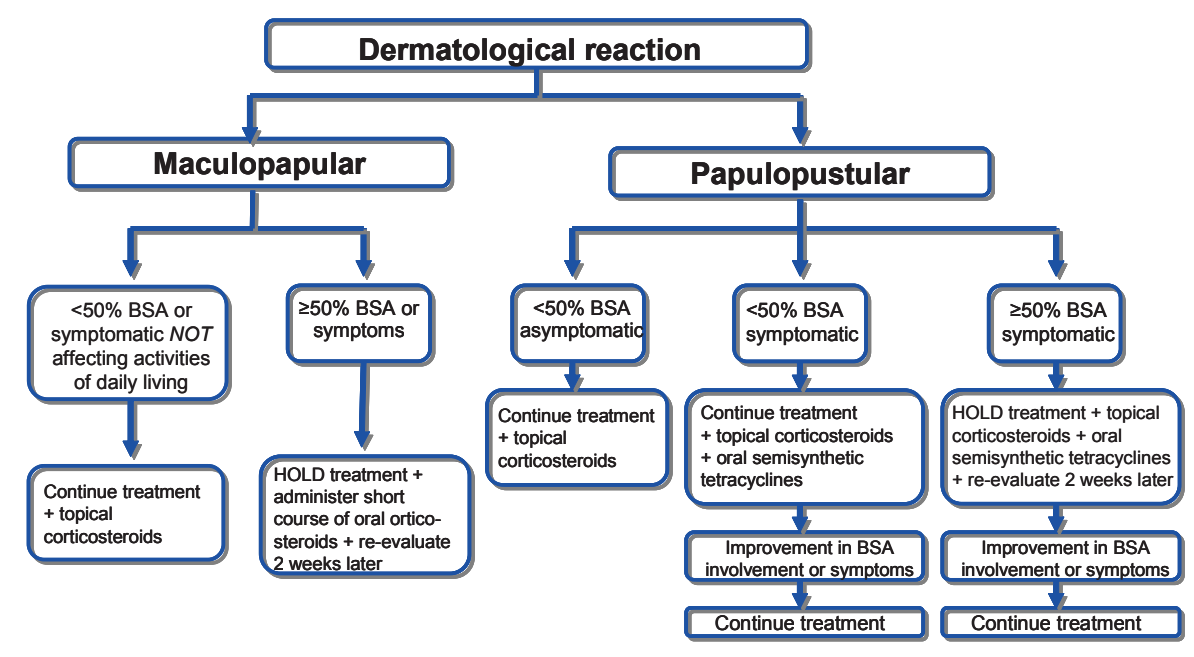

teroids or tetracyclines (e.g. minocycline) in more severe cases. Lapatinib should only be discontinued if more than $50 \%$ of the body surface is affected. A re-evaluation of the patient is indicated after 2 weeks, and treatment can be resumed in case of amelioration of the skin eruptions $[9,10]$. A detailed algorithm for the treatment of skin reactions is shown in figure 2.

\section{Cardiotoxicity of Anti-ErbB2 Therapies}

Cardiotoxicity is a well established adverse effect of antitumor therapies, especially of anthracyclines. Of prognostic importance is the dose-dependent development of congestive heart failure (CHF) in anthracycline-treated patients due to myocardial cell death and consecutive irreversible damage to the heart. Anti-ErbB2-targeted cancer drugs can worsen anthracycline-associated cardiotoxicity, particularly when given concomitantly, because they inhibit a cardioprotective mechanism [11]. In clinical trials, treatment with the monoclonal anti-ErbB2 antibody trastuzumab in combination with chemotherapy was associated with the development of cardiac dysfunction in kip to $28 \%$ of patients $[12,13]$. In contrast to anthracyclines which (depending on the dose) can induce apoptosis and necrosis of myocytes and therefore induce irreversible cardiac damage, therapies directed against the ErbB2 pathway used as single agents predominantly cause reversible contractile dysfunction.

The precise mechanism of cardiac dysfunction during antiErbB2 treatment is not understood in full detail. The ErbB2 signaling pathway in cardiomyocytes is thought to be essential to normal cardiac function and to protect cardiomyocytes from damage induced by hypoxia or oxidative stress [11, 14, 15]. While the interruption of ErbB2 signaling due to trastuzumab or lapatinib monotherapy appears to be tolerated by normal cardiomyocytes, this may change in a setting of already increased oxidative stress induced by anthracyclines. Chien [16] proposed a two-hit molecular model for trastuzu- mab-associated cardiotoxicity, where the first hit may represent an activation of stress signaling pathways by anthracyclines. This can be overcome by neuregulin binding to ErbB2/ErbB4 heterodimers and stimulating cardiomyocyte survival pathways. By blocking ErbB2, trastuzumab may interrupt these pathways, thus delivering the second hit ultimately leading to cell dysfunction.

The rate of trastuzumab-associated cardiotoxicity in the recently published adjuvant trials in patients with ErbB2-overexpressing breast cancer appears to be lower than in the pivotal trials in metastastic disease and ranged from 0 to $4 \%$ induction of severe heart failure and 3 to $18 \%$ contractile dysfunction [17-22]. Due to different trial designs with different inclusion criteria and not identical definitions of cardiac events, it is difficult to make comparisons between the trials. However, it appears that the use of anthracyclines prior to trastuzumab, a short time period between the end of chemotherapy and the initiation of anti-ErbB2 treatments, and possibly the combination of trastuzumab with paclitaxel may increase the risk of trastuzumab-associated cardiotoxicity [21]. In this context, the cardiotoxicity rate of lapatinib so far appears to be in an acceptable range. Perez et al. [23] recently reported a combined incidence of $\mathrm{CHF}$ and asymptomatic systolic dysfunction of $1.8 \%$ in over 3,000 patients treated with lapatinib. However, it is important to point out that the treatment strategies in these patients were significantly different from those in the adjuvant trastuzumab trials. Lapatinib is now being studied in the ALTTO trial as adjuvant therapy, and the design of the trial will allow to better assess the risk of lapatinib-associated cardiotoxicity. Based on the relatively low incidence of cardiotoxicity in the pivotal trials, it was decided to accept a left ventricular ejection fraction (LVEF) of $50 \%$ or higher as eligibility criterion for the inclusion of patients. The same threshold was used in NSABP B-31, NCCTG N 9831, and BCIRG 006 adjuvant trastuzumab trials, whereas the HERA trial required a LVEF of $55 \%$ or higher. 


\section{Assessment of Cardiac Risk in Breast Cancer Patients Receiving Anti-ErbB2 Therapies}

Prior to starting treatment with anti-ErbB2 therapies, baseline LVEF should be checked in every patient by multiple-gated acquisition (MUGA) scan or echocardiography. Patients with systolic dysfunction (i.e. LVEF $<50 \%$ ) are not good candidates for anti-ErbB2 therapy since they have a higher risk of treatment-associates cardiotoxicity [22]. LVEF measurements should be repeated once or twice during therapy or if clinically indicated, and at the end of treatment. In the case of an asymptomatic drop in LVEF, a repeat LVEF assessment should be performed after 3-4 weeks, and treatment should be held if the LVEF is lower than $40 \%$; the patient should then be referred to a cardiologist. In addition, measurements of cardiac biomarkers (creatinin kinase, troponin, brain natriuretic peptide) are strongly recommended in order to detect myocardial cell loss, a condition that is likely more serious than systolic dysfunction without increased cardiac biomarkers. Again, in patients with systolic dysfunction and increased cardiac biomarkers, it is strongly recommended to consult a cardiologist and to begin treatment for systolic dysfunction.

\section{Patient Information and Shared Decision-Making}

With all oral anti-cancer therapies, good compliance and high treatment adhesion rates are critical to achieve treatment goals. If patients are not convinced of the efficacy of a therapy or are worried about potential side effects of a drug, compliance will be suboptimal, even in the case of breast cancer as a potentially life-threatening disease. In this context, a good relationship between patient and physician is essential. The cornerstone of such a relationship should be ample information about the nature of the disease, the current knowledge in the field of research, possible treatment options, their mode of action, and their risk/benefit ratio. Physicians should be aware that especially in the case of aggressive ErbB2-positive tumors, most breast cancer patients are extremely worried and anxious. These feelings should be addressed in the conversation with the patient to enable the participation of the patient in treatment decisions ('shared decision-making') even in this difficult situation. This includes information about the availability of modern drugs against this type of tumor. Talking with other patients with ErbB2-positive breast cancer, who courageously and successfully fight against their disease, can be reassuring for a newly diagnosed patient. A well informed patient can be demanding for the physician. But this patient will probably be more satisfied with her treatment and will hopefully be more compliant, which generally leads to improved treatment outcome.

\section{References}

$>1$ Liu X, Gu X, Li Z, et al.: Neuregulin-1/erbB activation improves cardiac function and survival in models of ischemic, dilated and viral cardiomyopathy. $\mathrm{J}$ Am Coll Cardiol 2006;48:1438-1447.

2 Ylä-Herttuala S, Rissannen TT, Vajanto I, et al.: Vascular endothelial growth factors: biology and current status of clinical application in cardiovascular medicine. J Am Coll Cardiol 2007;49:1015-1026.

$\checkmark 3$ Geyer CE, Forster J, Lindquist D, et al.: Lapatinib plus capecitabine for HER2-positive advanced breast cancer. N Engl J Med 2006;355:2733-2743.

4 Di Leo A, Gomez H, Aziz Z, et al.: Lapatinib plus paclitaxel compared to paclitaxel as first-line therapy in patients with metastatic breast cancer: a phase III randomized double-blind study of 580 patients. Proc Am Soc Clin Oncol 2007;25(18S): abstr 1011.

5 Jones SF, Burris HA, Yardley DA, et al.: Lapatinib (an oral dual kinase inhibitor) plus weekly or every 3 week paclitaxel. Proc SABCS 2004, abstr 1069.

6 Koehler M, Chan S, Newstat BO, et al.: Diarrhea events in cancer patients treated with lapatinib. Proc Am Soc Clin Oncol 2007;25(18S):abstr 9125.

7 Benson A, Ajani A, Catalano RB, et al.: Recommended guidelines for the treatment of cancer treatment-induced diarrhea. J Clin Oncol 2004;22: 2918-2926.

8 Sweetman R, Lacouture ME, Koehler M, et al.: Skin events among 1126 patients treated with lapatinib, an oral dual ErbB1/2 tyrosine kinase inhibitor. Proc Am Soc Clin Oncol 2007;25(18S):abstr 9102.
9 Lacouture ME: Mechanisms of cutaneous toxicities to EGFR inhibitors. Nat Rev Cancer 2006;6:803812 .

10 Moy B, Goss P: Lapatinib-associated toxicity and practical management recommendations. Oncologist 2007:12:756-765

11 Timolati F, Ott D, Pentassuglia L, et al.: Neuregulin1 beta attenuates doxorubicin-induced alterations. J Mol Cell Cardiol 2006:41:845-854.

12 Seidman A, Hudis C, Pierri MK, et al.: Cardiac dysfunction in the trastuzumab clinical trials experience. J Clin Oncol 2002;20:1215-1221.

13 Suter TM, Cook-Bruns N, Barton C: Cardiotoxicity associated with trastuzumab (Herceptin) therapy in the treatment of metastatic breast cancer. Breast 2004:13:173-183.

14 Sawyer DB, Zuppinger C, Miller TA, et al.: Modulation of anthracycline-induced myofibrillar disarray in rat ventricular myocytes by neuregulin-1ß and anti-erbB2: potential mechanisms for trastuzumab-induced cardiotoxicity. Circulation 2002;105: 1551-1554.

15 Zuppinger C, Timolati F, Suter TM: Pathophysiology and diagnosis of cancer drug induced cardiomyopathy. Cardiovasc Toxicol 2007;7:61-66.

16 Chien KR: Stress pathways and heart failure. Cell 1999;98:555-558.

17 Romond EH, Perez EA, Bryant J, et al.: Trastuzumab plus adjuvant chemotherapy for operable HER2-positive breast cancer. N Engl J Med 2005; 353:1673-1684.
18 Slamon D, Eiermann W, Robert N, et al.: Phase III randomized trial comparing doxorubicin and cyclophosphamide followed by docetaxel (ACT) with doxorubicin and cyclophosphamide followed by docetaxel and trastuzumab (ACTH) with docetaxel, carboplatin and trastuzumab (TCH) in HER2 positive early breast cancer patients. BCIRG 006 study. Breast Cancer Res Treat 2005;94(suppl 1):S5a.

19 Piccart-Gebhart MJ, Procter M, Leyland-Jones B, et al.: Trastuzumab after adjuvant therapy in HER2positive breast cancer. N Engl J Med 2005;353: 1659-1672.

20 Joensuu H, Kellokumpu-Leptinen PL, Bono P, et al.: Adjuvant docetaxel or vinorelbin with or without trastuzumab for breast cancer. N Engl J Med 2006;354:809-820.

21 Suter TM, Procter M, van Veldhuisen DJ, et al. Trastuzumab-associated cardiac adverse effects in the herceptin adjuvant trial. J Clin Oncol 2007;25: 3859-3865.

22 Tan-Chiu E, Yothers G, Romond E, et al.: Assessment of cardiac dysfunction in a randomized trial comparing doxorubicin and cyclophosphamide followed by paclitaxel, with or without trastuzumab as adjuvant therapy in node-positive, human epidermal growth factor receptor-2 overexpressing breast cancer: NSABP-B-31. J Clin Oncol 2005;23:78117819.

23 Perez EA, Byrne JA, Isaac W, et al.: Cardiac safety experience in 3127 patients treated with lapatinib. Ann Oncol 2006;17(suppl 9):ix70. 